\title{
APLICAÇÃO DO CONTROLADOR LQR EM MANOBRAS DE RENDEZVOUS PARA SATÉLITE ALVO EM ÓRBITA LEO
}

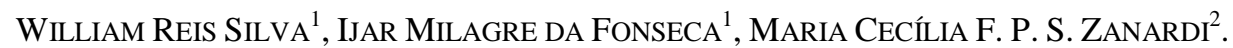 \\ 1. Divisão de Mecânica Espacial e Controle, Instituto Nacional de Pesquisas Espaciais (INPE) \\ Av dos Astronautas, 1758, Jd. da Granja, CEP:12227-010, São José dos Campos, São Paulo, Brasil. \\ E-mails: reis.williamegmail.com, ijaredem.inpe.br \\ 2. Grupo de Dinâmica Orbital e Planetologia, Universidade Estadual Paulista (UNESP) \\ Av. Ariberto Pereira da Cunha, 333, Pedregulho, CEP:12516-410 \\ E-mail: cecilia@feg.unesp.br
}

\begin{abstract}
This work aims to realize a study of rendezvous maneuvers between satellite atmospheric reentry (SARA), regarded as a chaser vehicle, and a target vehicle in orbit LEO permanent. A theoretical study of the dynamic equations for the relative motion, proposed by Hill-Clohessy-Wiltshire, and a study of control system for such rendezvous maneuver is performed, considering the dynamics of the actuators negligible. The control technique of multivariable Linear Quadratic Regulator (LQR) is used as a method to design the control system making use of the computational tool MATLAB. In simulations of rendezvous maneuvers, it was used as a strategy, approaches between the chaser and target vehicles by V-bar and its was analyzed the temporal evolution of the position, velocity and the force required to the control with the intention to evaluate the response speed of such control system. With the graphical visualization of 3D maneuvers, it is observed that the LQR control technique applied to the problem was adequate and with satisfactory results. Stresses those, to design, understand and manipulate controllers for orbital maneuvers of rendezvous accurate can make the difference between success or failure of a project that certainly involves high budget.
\end{abstract}

Keywords— Orbital Dynamics, Rendezvous, LQR Controller, Hill-Clohessy-Wiltshire Equation.

\begin{abstract}
Resumo - Este trabalho tem por objetivo realizar um estudo de manobras de rendezvous entre o Satélite de Reentrada Atmosférica (SARA), considerado como um veículo perseguidor, e um veículo alvo em órbita permanente LEO. Um estudo teórico da modelagem das equações dinâmicas de movimento relativo, propostas por Hill-Clohessy-Wiltshire, e um estudo do sistema de controle para tais manobras de rendezvous é realizado, considerando a dinâmica dos atuadores desprezíveis. A técnica de controle de multivariáveis Regulador Linear Quadrático (LQR) é utilizada como método para projetar o sistema de controle fazendo uso da ferramenta computacional MATLAB. Nas simulações das manobras de rendezvous, utilizou-se como estratégia, aproximações entre os veículos perseguidor e alvo por $V$-bar e são analisadas a evolução temporal da posição, velocidade e da força necessária para o controle com a intenção de avaliar a velocidade de resposta de tal sistema de controle. Com a visualização gráfica das manobras em 3D, observa-se que a técnica de controle LQR aplicada à planta do problema mostrou-se adequada e com resultados satisfatórios. Salienta-se que projetar, entender e manipular controladores cada vez mais precisos para manobras orbitais de rendezvous pode fazer a diferença entre o sucesso ou fracasso de um projeto que certamente envolve alto orçamento.
\end{abstract}

Palavras-chave— Dinâmica Orbital, Rendezvous, Controlador LQR, Equação de Hill-Clohessy-Wiltshire.

\section{Introdução}

Recentemente, devido ao alto risco e custo de se enviar astronautas ao espaço, uma nova tendência vem surgindo e sendo implementada para se realizar operações de rendezvous (encontro) e docking (acoplamento). Estas operações estão migrando para serem executadas de forma totalmente autônoma, ou seja, sem a interferência humana in-loco. Porém, para se desenvolver sistemas com tal nível de automatização, deve-se levar em consideração a necessidade de tecnologias inovadoras, precisas e robustas.

O procedimento de rendezvous e docking consiste em uma série de manobras orbitais e trajetórias controladas, que sucessivamente levam um veículo ativo (perseguidor) até a vizinhança e gradativamente até o contato com um veículo passivo (alvo).

$\mathrm{O}$ processo de rendezvous refere-se às manobras orbitais responsáveis por alinhar o voo de duas espaçonaves, sincronizando seus elementos orbitais e reunindo-as no mesmo plano orbital. Ao final desta operação, ambos os veículos estarão emparelhados.
O veículo perseguidor deve chegar para se acoplar com o alvo com velocidades relativas lineares e angulares nulas ou próximas de zero. Para que isso seja possível, além de reduzir sua velocidade de aproximação, o perseguidor deve também sincronizar sua atitude com a do alvo (Gentina, 2009).

Neste trabalho as equações de movimento relativo entre o veículo perseguidor e o veículo alvo são descritas pelas equações de Hill-Clohessy-Wiltshire, em que considera-se o veículo alvo em uma órbita circular. O método de controle utilizado para as manobras de rendezvous é o Regulado Linear Quadrático (LQR).

\section{A Equação de Hill-Clohessy-Wiltshire}

A metodologia utilizada para o cálculo de guiamento do veículo perseguidor durante as manobras de rendezvous são introduzidas por Clohessy-Wiltshire (1960) em que é considerado um sistema de eixos de referência composto da seguinte forma: sua origem é centralizada no centro de massa do veículo alvo para 
que todas as outras coordenadas sejam representadas em relação a este veículo. Dessa forma, quando o veículo perseguidor estiver na origem do sistema de referência (ao passo de um determinado offset deste para que não haja colisões) ele terá efetivado a manobra de acoplamento.

Tal sistema de coordenadas está representado em vermelho na Figura 1 a seguir.

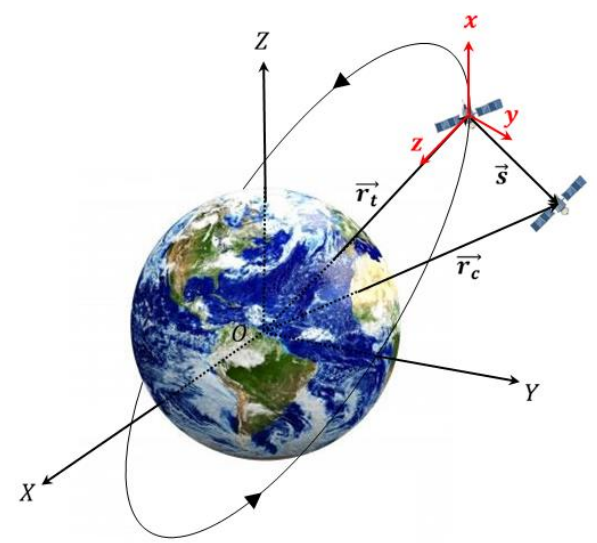

Figura 1. Órbitas do veículo perseguidor e veículo alvo. Sistema de referência centralizado no veículo alvo.

Além de seu centro estar contido no satélite alvo e seu plano de referência estar no plano do horizonte local deste veículo, seus eixos possuem a seguinte orientação:

- Eixo x: na mesma direção e sentindo do vetor velocidade orbital $(V$-bar $)$;

- Eixo y: normal à órbita, com sentido oposto ao vetor momento angular orbital (H-bar);

- Eixo z: completa o sistema dextrógiro, orientado na direção radial, perpendicular ao plano do horizonte, sentido nadir $(R$-bar).

Sabendo que, $\overrightarrow{r_{t}}$ e $\overrightarrow{r_{c}}$ são as distâncias do centro de massa da Terra até os veículos, alvo (target) e perseguidor (chaser) respectivamente e $\vec{s}$ a distância relativa entre os veículos.

$$
\vec{s}=\overrightarrow{r_{c}}-\overrightarrow{r_{t}}
$$

A equação geral de movimento de corpo sobre influência da força central é dada pela lei da gravitação de Newton (1713).

$$
\begin{aligned}
& m_{c} \ddot{\overrightarrow{r_{c}}}=-\mu \frac{m_{c}}{r_{c}{ }^{3}} \overrightarrow{r_{c}}+\vec{F}_{c n g} \\
& m_{t} \ddot{\overrightarrow{r_{t}}}=-\mu \frac{m_{t}}{r_{t}{ }^{3}} \overrightarrow{r_{t}}+\vec{F}_{t n g}
\end{aligned}
$$

sendo, $m_{c}$ e $m_{t}$ as massas dos veículos, perseguidor e alvo, respectivamente; $\vec{F}_{c n g}$ e $\vec{F}_{t n g}$ são forças não gravitacionais nos veículos, perseguidor e alvo, respectivamente. Essas forças incluem perturbações externas e a atuação do controle.
Substituindo as Equações (2) e (3) na derivada de segunda ordem da Equação (1), considerando apenas que o controle é realizado somente pelo sistema propulsivo presente no veículo perseguidor e desconsiderando a influência de perturbação externa no sistema $\left(\vec{F}_{t n g}=\overrightarrow{0}\right.$ e $\left.\vec{F}_{c n g}=\overrightarrow{u_{c}}\right)$, obtém-se:

$$
\ddot{\vec{s}}=-\frac{\mu}{r_{c}{ }^{3}} \overrightarrow{r_{c}}+\frac{\mu}{r_{t}{ }^{3}} \overrightarrow{r_{t}}+\frac{\overrightarrow{u_{c}}}{m_{c}}
$$

Linearizando a força gravitacional no veículo perseguidor ao redor da posição do veículo alvo por meio de uma expansão em série de Taylor até a primeira ordem, obtém-se (Fehse, 2003).

$$
\ddot{\vec{s}}=-\frac{\mu}{r_{c}{ }^{3}} M \vec{s}+\frac{\overrightarrow{u_{c}}}{m_{c}}
$$

em que

$$
M=\left[\begin{array}{ccc}
1-3 \frac{r_{x}{ }^{2}}{r_{t}{ }^{2}} & -3 \frac{r_{x} r_{y}}{r_{t}{ }^{2}} & -3 \frac{r_{x} r_{z}}{r_{t}{ }^{2}} \\
-3 \frac{r_{x} r_{y}}{r_{t}{ }^{2}} & 1-3 \frac{r_{y}{ }^{2}}{r_{t}{ }^{2}} & -3 \frac{r_{y} r_{z}}{r_{t}{ }^{2}} \\
-3 \frac{r_{x} r_{z}}{r_{t}{ }^{2}} & -3 \frac{r_{z} r_{y}}{r_{t}{ }^{2}} & 1-3 \frac{r_{z}{ }^{2}}{r_{t}{ }^{2}}
\end{array}\right]
$$

O objetivo é representar o movimento do veículo perseguidor no referencial no veículo alvo, porém este é um referencial não inercial. Assim esta relação é dada por (Lemos, 2007):

$$
\ddot{\vec{s}}=\ddot{\overrightarrow{s^{*}}}+\vec{\omega} \times\left(\vec{\omega} \times \overrightarrow{s^{*}}\right)+2 \vec{\omega} \times \dot{\overrightarrow{s^{*}}}+\dot{\vec{\omega}} \times \overrightarrow{s^{*}}
$$

Substituindo a Equação (7) na Equação (5), obtém-se:

$$
\ddot{\overrightarrow{s^{*}}}+\vec{\omega} \times\left(\vec{\omega} \times \overrightarrow{s^{*}}\right)+2 \vec{\omega} \times \dot{\overrightarrow{s^{*}}}+\dot{\vec{\omega}} \times \overrightarrow{s^{*}}=-\frac{\mu}{r_{c}{ }^{3}} M \vec{s}+\frac{\overrightarrow{u_{c}}}{m_{c}}
$$

Sabendo que $\overrightarrow{s^{*}}=\left[\begin{array}{lll}x & y & z\end{array}\right]^{T}$ e representando $\vec{\omega}$ e $\overrightarrow{r_{t}}$ em VHR-bar como:

$$
\begin{aligned}
& \vec{\omega}=\left[\begin{array}{c}
0 \\
-\omega \\
0
\end{array}\right] \\
& \overrightarrow{r_{t}}=\left[\begin{array}{l}
0 \\
0 \\
r_{t}
\end{array}\right]
\end{aligned}
$$

Substituindo as Equações (9) e (10) na Equação (8) e evoluindo os cálculos, obtêm-se as equações de movimento do problema (Junior, 2011):

$$
\begin{gathered}
\ddot{x}=2 \omega \dot{z}+\omega^{2} x+\dot{\omega} z-\frac{\mu}{r_{t}{ }^{3}} x+\frac{1}{m_{c}} u_{x} \\
\ddot{y}=-\frac{\mu}{r_{t}{ }^{3}} y+\frac{1}{m_{c}} u_{y} \\
\ddot{z}=-2 \omega \dot{x}+\omega^{2} z-\dot{\omega} x+2 \frac{\mu}{r_{t}{ }^{3}} z+\frac{1}{m_{c}} u_{z}
\end{gathered}
$$


Para o caso especial em que o veículo alvo se encontra em uma órbita circular $r_{t}$ e $\omega$ são constantes, dessa forma, de acordo com Bate et. al. (1971).

$$
\omega^{2}=\frac{\mu}{r_{t}^{3}}
$$

Logo, usando a Equação (14) nas Equações (11), (12) e (13) as equações de movimento se reduzem em uma forma simples conhecida com Equações de Hill-Clohessy-Wiltshire.

$$
\begin{gathered}
\ddot{x}=2 \omega \dot{z}+\frac{1}{m_{c}} u_{x} \\
\ddot{y}=-\omega^{2} y+\frac{1}{m_{c}} u_{y} \\
\ddot{z}=-2 \omega \dot{x}+3 \omega^{2} z+\frac{1}{m_{c}} u_{z}
\end{gathered}
$$

Essas serão as equações de movimento utilizadas nesse trabalho, uma vez que a grande maioria das manobras de rendezvous é realizada com o veículo alvo e órbita circular.

É importante notar que, as Equações (15) e (17) estão acopladas entre si, dessa forma será conveniente separar os movimentos em: fora do plano orbital $H$-bar (componente $y$ ) e no plano orbital $R V$-bar (componentes $x z$ ).

Assim, para a estratégia de controle separa-se o sistema em dois subsistemas, sendo um SISO ( $H$ bar) e outro MIMO (RV-bar).

\section{Representação da Planta no Espaço de Estados}

\subsection{Modelo SISO para movimento em H-bar}

Aqui o subsistema se apresenta com dois estados, contendo uma entrada e uma saída.

$$
\begin{gathered}
{\left[\begin{array}{l}
\dot{x}_{1} \\
\dot{x}_{2}
\end{array}\right]=\left[\begin{array}{cc}
0 & 1 \\
-\omega^{2} & 0
\end{array}\right]\left[\begin{array}{l}
x_{1} \\
x_{2}
\end{array}\right]+\left[\begin{array}{c}
0 \\
1 \\
m_{c}
\end{array}\right] u_{y}} \\
y=\left[\begin{array}{ll}
1 & 0
\end{array}\right]\left[\begin{array}{l}
x_{1} \\
x_{2}
\end{array}\right]
\end{gathered}
$$

em que $x_{1}$ e $x_{2}$ representam a posição $y$ e a velocidade $\dot{y}$, respectivamente.

\subsection{Modelo SISO para movimento em RV-bar}

Aqui o subsistema se apresenta com quatro estados, contendo duas entradas e duas saídas.

$$
\left[\begin{array}{l}
\dot{x}_{3} \\
\dot{x}_{4} \\
\dot{x}_{5} \\
\dot{x}_{6}
\end{array}\right]=\left[\begin{array}{cccc}
0 & 0 & 1 & 0 \\
0 & 0 & 0 & 1 \\
0 & 0 & 0 & 2 \omega \\
0 & 3 \omega^{2} & -2 \omega & 0
\end{array}\right]\left[\begin{array}{l}
x_{3} \\
x_{4} \\
x_{5} \\
x_{6}
\end{array}\right]+\left[\begin{array}{cc}
0 & 0 \\
0 & 0 \\
\frac{1}{m_{c}} & 0 \\
0 & \frac{1}{m_{c}}
\end{array}\right]\left[\begin{array}{l}
u_{y} \\
u_{y}
\end{array}\right]
$$

$$
y=\left[\begin{array}{llll}
1 & 0 & 0 & 0 \\
0 & 1 & 0 & 0
\end{array}\right]\left[\begin{array}{l}
x_{3} \\
x_{4} \\
x_{5} \\
x_{6}
\end{array}\right]
$$

em que $x_{3}, x_{4}, x_{5}$ e $x_{6}$ representam as posições $x, z$ as velocidades $\dot{x}$ e $\dot{z}$, respectivamente.

\section{Dados Utilizados}

Nessa seção são apresentadas as características físicas e orbitais do satélite perseguidor, tais dados são utilizados nas simulações realizadas neste trabalho.

\subsection{Plataforma orbital recuperável: SARA}

O Satélite de Reentrada Atmosférica (SARA) trata-se de uma Plataforma Orbital Recuperável de aplicações científica de aproximadamente $350 \mathrm{~kg}$ de mas$\mathrm{sa}$, atualmente em desenvolvimento no Instituto de Aeronáutica e Espaço (IAE), do CTA, em São José dos Campos, SP (Junior, 2008).

Este satélite será lançado a uma órbita baixa terrestre (LEO) de $300 \mathrm{~km}$ de altitude em órbita equatorial e aproximadamente após 10 dias em órbita a espaçonave irá realizar uma manobra de reentrada atmosférica controlada. Após a reentrada, o veículo deverá ser recuperado para posterior reutilização do seu módulo reentrável (Seito, 2007).

O SARA está sendo projetado para fins de aplicações científicas e será constituído por um veículo com uma carga útil de $55 \mathrm{~kg}$ contendo pequenos experimentos científicos e tecnológicos. Alguns destes experimentos poderão necessitar ser atendidos ou servidos em órbita para serem bem-sucedidos ao longo do tempo de duração da missão

Dessa forma, o SARA deverá incorporar dispositivos de acoplamento que serão utilizados por outro segmento orbital permanente (veículo alvo). Sendo assim, o perseguidor deverá executar uma série de operações de rendezvous e docking a fim de completar os objetivos da missão.

As configurações do SARA são apresentadas na Tabela 1 e 2 a seguir.

Tabela 1. Parâmetro físico do Satélite de Reentrada Atmosférica (SARA).

\begin{tabular}{cc}
\hline Característica física & Valores \\
\hline Massa $\left(m_{c}\right)$ & $350 \mathrm{~kg}$ \\
\hline
\end{tabular}

Tabela 2. Parâmetros orbitais do Satélite de Reentrada Atmosférica (SARA).

\begin{tabular}{cc}
\hline Parâmetros orbitais & Valores \\
\hline Altitude $(h)$ & $300 \mathrm{~km}$ \\
Excentricidade $(e)$ & $\cong 0$ \\
Inclinação $(I)$ & $0^{\circ}$ \\
Velocidade angular $(\omega)$ & $1,16 \times 10^{-3} \mathrm{rad} / \mathrm{s}$ \\
\hline
\end{tabular}




\section{Método de Controle}

A técnica de controle utilizada neste trabalho para projetar o controlador ótimo para a manobra de rendezvous é o Regulador Linear Quadrático (LQR). Tal técnica será usada com auxílio da ferramenta computacional $M A T L A B^{\circledR}$.

\subsection{Regulador Linear Quadrático}

No caso do Regulador Linear Quadrático (LQR), assume-se que todos os estados estão disponíveis para realimentação (Pinheiro, 2012). Considere a dinâmica do sistema dada por:

$$
\begin{gathered}
\dot{x}(t)=A x(t)+B u(t) \\
y(t)=C x(t)
\end{gathered}
$$

sendo $A$ uma matriz $n \times n, B$ uma matriz $n \times m, C$ uma matriz $k \times n, x(t)$ as variáveis de estado $n \times 1$ e $u(t)$ o sinal de controle $m \times 1$.

Para resolver este problema de regulação do sinal de saída deve-se otimizar o custo funcional definido como:

$$
J=\int_{t_{0}}^{\infty}\left(x^{\prime} Q x+u^{\prime} R u\right) d t
$$

em que, $Q$ e $R$ são uma matrizes semi-definidas positivas, simétricas reais e geralmente diagonal.

Se $Q$ for maior que a $R$, a resposta do sistema melhora, porém a energia exercida pelo controle $u$ possivelmente aumentará. Se $R$ for maior que a $Q$, a resposta do sistema piora, pois a energia exercida pelo controle $u$ é mais penalizada.

Usando o princípio de Pontriagin (Kirk, 2004), pode-se escrever a Hamiltoniana do sistema como:

$$
H=\frac{1}{2}\left(x^{\prime} Q x+u^{\prime} R u\right)+\lambda^{\prime}(A x+B u)
$$

O princípio mínimo estabelece que o controle ótimo e as trajetórias dos estados devem satisfazer as seguintes três condições: a equação de estado, a equação de co-estado e a equação estacionária (Kirk, 2004).

Evoluindo os cálculos, encontra-se as seguintes equações:

$$
\begin{aligned}
\dot{x} & =A x+B u \\
-\dot{\lambda} & =Q x+A^{\prime} \lambda \\
u^{*} & =-R^{-1} B^{\prime} \lambda
\end{aligned}
$$

em que $u^{*}$ é o sinal de controle ótimo.

O problema de valor de contorno não precisa ser necessariamente resolvido, dessa forma, considera-se que.

$$
\lambda=P(t) x
$$

A formulação apresentada para a solução do LQR é conhecida como problema de tempo infinito que resulta em um controlador com retroação de estados linear variante no tempo, dado por:

$$
u(t)=-K(t) x(t)
$$

em que

$$
K(t)=R^{-1} B^{\prime} P(t)
$$

Na situação em que o processo a ser controlado reside em um intervalo de tempo infinito fornece uma solução simples para encontrar o controlador LQR (Kirk, 2004). Se o par $(A, B)$ é controlável, o par $(A, C)$ é observável e as matrizes $A, B, R$ e $Q$ são constantes, quando $t_{f}$ tende ao infinito, tem-se que $P(t)$ e $K(t)$ se tornar matrizes constantes $P$ e $K$.

Substituindo a Equação (29) na Equação (22) tem-se o controle da dinâmica em malha fechada.

$$
\begin{gathered}
\dot{x}(t)=(A-B K) x(t) \\
y(t)=C x(t)
\end{gathered}
$$

\section{Resultados Obtidos}

Nesta seção, serão apresentados os resultados obtidos para o controlador LQR com simulações da planta usando a ferramenta $M A T L A B^{\circledR}$. O sistema foi dividido em dois subsistemas, um MIMO, representando o movimento em $R V$-bar e outro SISO, representando o movimento em $H$-bar.

Nas simulações considerou-se que o veículo alvo encontrava-se em uma órbita circular permanente LEO e que o veículo perseguidor deveria realizar manobras de rendezvous se aproximando do veículo alvo por $V$-bar.

\subsection{Controlador LQR para o sistema SISO}

Os estados deste espaço são: a posição $(y)$ e a velocidade $(\dot{y})$. O sinal de controle $\left(u_{y}\right)$ é uma força aplicada ao sistema em $y$ e $\dot{y}$. A saída do sistema é a própria posição final.

Para validar o controlador, aplicou-se uma situação fíctícia ao satélite SARA, o veículo perseguidor, sendo que este deve realizar uma manobra de rendezvous até o veículo alvo. A Tabela 3, a seguir, apresenta a posição e velocidade iniciais em $\mathrm{H}$-bar do veículo perseguidor.

Tabela 3. Posição e velocidade iniciais em $H$-bar do SARA em relação ao veículo alvo

\begin{tabular}{cc}
\hline Parâmetros & Valores \\
\hline$y_{0}$ & $5,175 \mathrm{~m}$ \\
$\dot{y}_{0}$ & $3,1 \mathrm{~m} / \mathrm{s}$ \\
\hline
\end{tabular}


A Figura 2, a seguir, apresenta a evolução temporal da posição, velocidade e da força de controle para o controlador LQR.
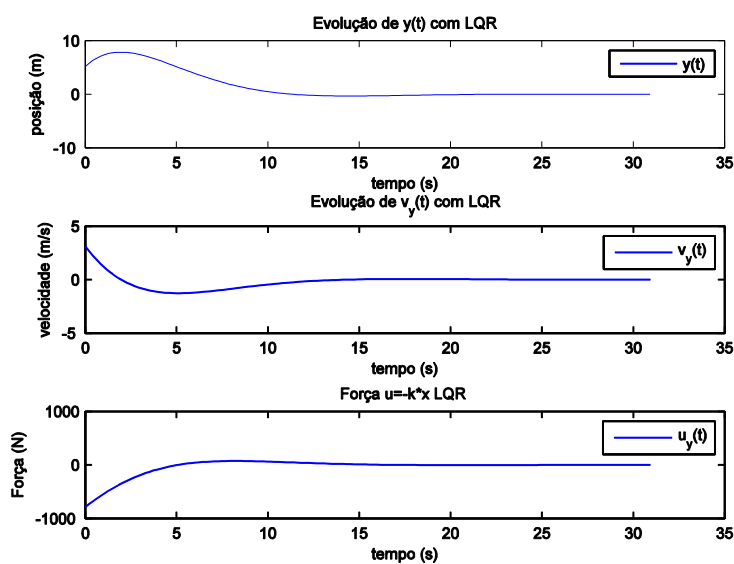

Figura 2. Comportamento da posição, velocidade e da força de controle em $H$-bar.

O veículo perseguidor alcançar um pico em $y$ de $7,82 \mathrm{~m}$ em $1,97 \mathrm{~s}$ e alcança a posição desejada $(y=0 m)$ e estabilizando em 21,22s. O pico de força exercida pelo controle é exatamente no início do processo, em módulo alcança $780 \mathrm{~N}$.

\subsection{Controlador LQR para o sistema MIMO}

Os estados deste espaço são: as posições $(x, z)$ e as velocidades $(\dot{x}, \dot{z})$.

Os sinais de controle $\left(u_{x}, u_{z}\right)$ são forças aplicadas ao sistema em $x, z, \dot{x}, \dot{z}$. A saída do sistema é a própria posição final.

Como feito anteriormente para o sistema SISO, para validar o controlador, aplicou-se uma situação fictícia ao satélite SARA, o veículo perseguidor, sendo que este deve realizar uma manobra de rendezvous até o veículo alvo por uma aproximação em $V$-bar.

A Tabela 4, a seguir, apresenta a posição e velocidade iniciais em $R V$-bar do veículo perseguidor.

Tabela 4. Posição e velocidade iniciais em $H$-bar do SARA em relação ao veículo alvo

\begin{tabular}{cc}
\hline Parâmetros & Valores \\
\hline$x_{0}$ & $11,175 \mathrm{~m}$ \\
$z_{0}$ & $0,305 \mathrm{~m}$ \\
$\dot{x}_{0}$ & $1,27 \mathrm{~m} / \mathrm{s}$ \\
$\dot{z}_{0}$ & $-2,05 \mathrm{~m} / \mathrm{s}$ \\
\hline
\end{tabular}

A Figura 3, a seguir, apresenta a evolução temporal da posição, velocidade e da força de controle para o controlador LQR.
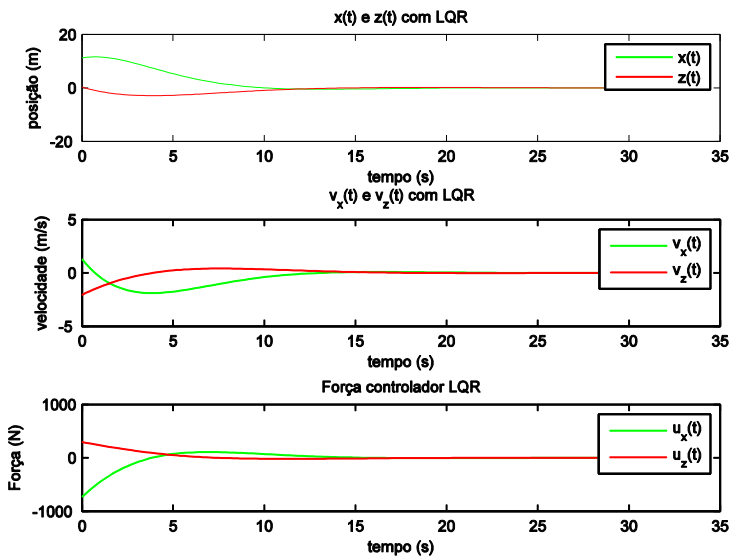

Figura 3 Comportamento da posição, velocidade e da força de controle em $R V$-bar.

$\mathrm{O}$ veículo perseguidor alcançar um pico em $x$ de $11,6 \mathrm{~m}$ em $0,83 \mathrm{~s}$ e um pico em $z$ de $-2,93 \mathrm{~m}$ em $3,94 s$, alcançando a posição desejada $(x=z=0 \mathrm{~m})$ e estabilizando em 25,53s. O pico de força exercida pelo controle está no início do processo, em módulo alcança $724,1 N$ no eixo $x$ e $291,7 N$ no eixo $z$.

As Figuras (4) e (5) representam o movimento do perseguidor em relação ao alvo em $R V$-bar e em $H$-bar versus $t(s)$, respectivamente.

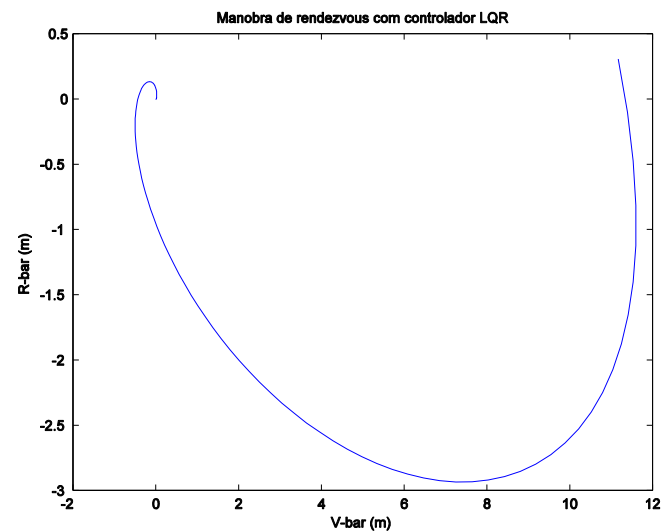

Figura 4. Movimento em $R V$-bar, aproximação por $V$-bar com controle LQR.

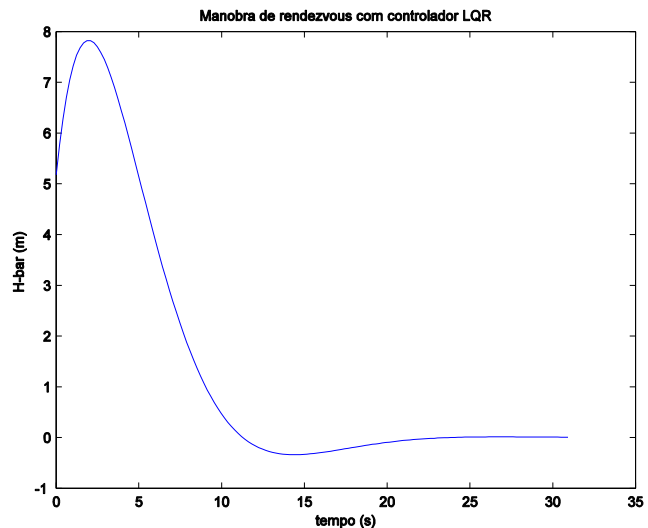

Figura 5. Movimento em $H$-bar versus t(s), aproximação em $V$-bar com controle LQR. 
Para facilitar a visualização, a Figura 6 representa o movimento do veículo perseguidor em relação ao veículo alvo em $V H R$-bar.

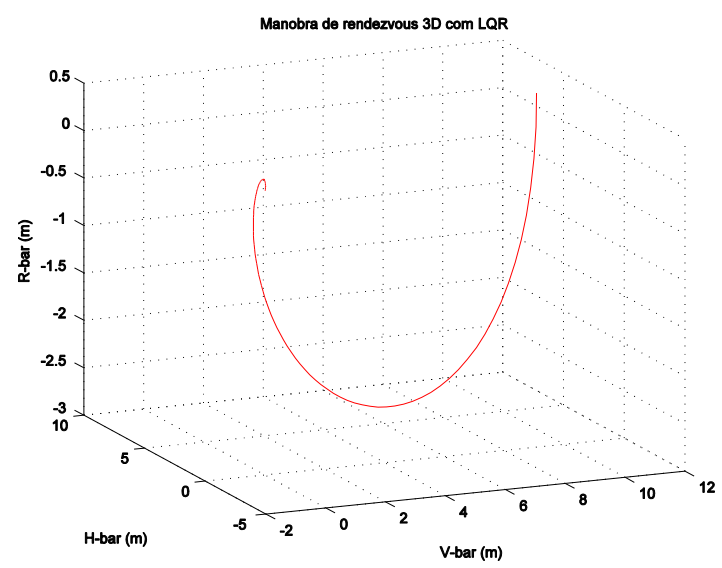

Figura 6. Movimento em $V H R$-bar com controle LQR, aproximação por $V$-bar.

\section{Conclusão}

Neste trabalho foi realizado um análise simulada de manobras de rendezvous entre o Satélite de Reentrada Atmosférica (SARA) em uma veículo alvo em uma órbita LEO com o controlador LQR.

A técnica de controle LQR aplicada na planta do problema, que foi separada em dois subsistemas sendo um SISO, para representar o movimento em $H$-bar e outro MIMO, para representar o movimento em $R V$-bar, apresentou resultados satisfatórios as possíveis especificações de projeto.

Para a manobra de rendezvous, simulou-se uma situação em que uma como veículo perseguidor se aproximando por $V$-bar do veículo alvo. Lembrando que a aproximação em $V$-bar é considerada a forma mais segura e em geral de menor consumo energético uma vez que, quando a espaçonave se aproxima do alvo pela "frente", ou seja, na direção do vetor velocidade, ela realiza "saltos" sucessivos na direção do alvo por meio do seu levantamento orbital.

Sendo assim, quando sua órbita aumenta, sua velocidade orbital diminui, aumentando assim sua velocidade relativa em relação ao alvo (em órbita inferior, portanto mais rápida). Isso faz com que o perseguidor se aproxime do alvo sucessivamente com baixas velocidades relativas, exigindo também um consumo menor de combustível.

A técnica de controle LQR é de fácil aplicação, tanto em sistemas SIMO como em sistemas MIMO, porém deve-se lembrar de que ela carrega algumas limitações. Tal técnica não leva em consideração ruídos dinâmicos e incertezas nas medidas dos estados, além disso, é necessário conhecer todos os esta- dos da planta para aplicá-lo, sendo necessário carregar o satélite perseguidor com sensores para medir posições e velocidades com relação ao veículo alvo.

\section{Agradecimentos}

Os autores gostariam de agradecer o suporte financeiro recebido pela CAPES e FAPESP (Proc. $\mathrm{N}^{\circ}$. 2012/21023-6)

\section{Referências Bibliográficas}

Bate, R. R.; Mueller, D. D.; White, J. E. (1971). Fundamentals of Astrodynamics. New York, USA: Dover. p. 455.

Clohessy, W. H.; Wiltshire, R. S. (1960). Terminal Guidance System for Satellite Rendezvous. Journal of the Aerospace Sciences, v. 27, n. 9, p. 653-659. $\quad$ DOI: $\quad 10.2514 / 8.8704$

Fehse, W. (2003). Automated Rendezvous and Docking of Spacecraft. New York, USA: Cambridge University Press. p. 517. DOI: 10.1017/CBO9780511543388

Gentina, J. (2009). Desenvolvimento e Simulação da Lógica Embarcada para Manobras de Rendezvous e Docking da Plataforma Orbital Recuperável SARA.. Dissertação de Mestrado, Instituto Nacional de Pesquisas Espaciais (INPE), São José dos Campos.

Junior, P. M. (2008). Design Aspects of the Recoverable Orbital Platform SARA. Chilean Congress of Mechanical Engineering, Unesp, Concepcion, p. 8.

Junior, G. A. (2011). Rendezvous with a Noncooperating Target. Thesis (PH.D) | Universitat Bremen, Bremen.

Kirk, D. E. (2004). Optimal Control Theory: An Introduction. New York, USA: Dover Publications. 3.ed.

Lemos, N. A. (2007). Mecânica Analítica. São Paulo, Brasil: Editora Livraria da Física, p. 386, 2.ed.

Newton, I. (1713). Mathematical Principles of Natural Philosophy.

Pinheiro, E. R. (2012). Aplicaçao do Controlador misto $\mathrm{H}_{2} / \mathrm{H}_{\infty}$ no Controle da Atitude de um Microssatélite na Presença de Incertezas Estruturadas e não Estruturadas, p. 60 p. Proposta de Dissertação de Mestrado, Instituto Nacional de Pesquisas Espaciais (INPE), São José dos Campos.

Seito, N.; Fonseca, I. M. da; Junior, P. M. (2007). Sara Orbital Autonomous Rendezvous and Docking: A Scientific and Technological Challenge. Brazilian Conference on Dynamics, Control and their Applications, Unesp, São José do Rio Preto. 